\title{
Optics with hyperbolic materials
}

\section{Takayama, Osamu; Laurynenka, Andrei}

\section{Published in:}

Journal of the Optical Society of America - B

Link to article, DOI:

10.1364/JOSAB.36.000F38

Publication date:

2019

Document Version

Peer reviewed version

Link back to DTU Orbit

\section{Citation (APA):}

Takayama, O., \& Laurynenka, A. (2019). Optics with hyperbolic materials. Journal of the Optical Society of America - B, 36(8), F38-F48. https://doi.org/10.1364/JOSAB.36.000F38

\section{General rights}

Copyright and moral rights for the publications made accessible in the public portal are retained by the authors and/or other copyright owners and it is a condition of accessing publications that users recognise and abide by the legal requirements associated with these rights.

- Users may download and print one copy of any publication from the public portal for the purpose of private study or research.

- You may not further distribute the material or use it for any profit-making activity or commercial gain

- You may freely distribute the URL identifying the publication in the public portal

If you believe that this document breaches copyright please contact us providing details, and we will remove access to the work immediately and investigate your claim. 


\title{
Optics with hyperbolic materials [Invited]
}

\author{
Osamu Takayama* and Andrei V. LaVRinenko \\ DTU Fotonik - Department of Photonics Engineering, Technical University of Denmark, Ørsteds Plads \\ 343, DK-2800 Kgs. Lyngby, Denmark \\ *otak@fotonik.dtu.dk
}

\begin{abstract}
A hyperbolic material (HM) is an optical material that exhibits a unique property of having anisotropy with simultaneously different signs of the permittivity tensor components. Such property leads to novel optical phenomena. HM can host highly localized bulk plasmon polaritons and directional surface waves with large wavevectors required in various applications in nano-guiding, sensing, and imaging. The research on HMs has been intensified in last decade thanks to progress in nanofabrication and advances with two-dimensional materials. This article reviews the recent developments in novel structures and materials with hyperbolic materials, such as metasurfaces, hypercrystals, waveguides, cavities. Furthermore, various applications that take the advantage of unique optical phenomena with HMs are covered, including negative refraction and photonic spin Hall effect for optical signal sorting, super-resolution, refractive index sensing, and mid-infrared vibrational spectroscopy.
\end{abstract}

(C) 2019 Optical Society of America

\section{Introduction}

Hyperbolic materials (HMs) or indefinite media are highly anisotropic optical materials whose permittivity tensors have diagonal components with simultaneously different signs. Such permittivity properties generate a hyperbolic type of dispersion as the isofrequency contours are hyperbolic surfaces in the wavevector space [1-3]. In other words, due to the presence of negative and positive permittivity tensor components, a typical HM behaves as a metal with negative permittivity for one polarization of light and as a dielectric for another. There are examples of naturally-occurring materials with hyperbolic dispersion, most of them are so-called two-dimensional materials like graphene, or dichalcogenides of transition metals [1,2]. In contrary to the natural HMs which exhibit hyperbolic dispersion in certain wavelengths ranges, the artificial structures can satisfy the claim for indefinite permittivity (or permeability) in any desired range. The latter are called hyperbolic metamaterials (HMMs) [4-9]. Typically, HMMs contain alternating deeply-subwavelength metal and dielectric features. Among basic HMMs configurations there are multilayer stacks with thin films of subwavelength thicknesses [10-16], shallow [17,18] and deep nanotrenches structures [19-21], and arrays of metal nanowires [22-28] as illustrated in Fig. 1. In most of the cases HMMs possess uniaxial anisotropy characterized by ordinary $\varepsilon_{o}$ and extraordinary $\varepsilon_{e}$ permittivities. By definition, $\varepsilon_{o} \varepsilon_{e}<0$. Considering inevitable losses, the inequality should be applied to the real parts of the permittivities.

The underlying features enabling unique optics with HMs appear due to different signs of permittivity principal values. They are: (i) extremely large degree of anisotropy $\Delta \varepsilon=\left|\varepsilon_{o}-\varepsilon_{e}\right|$ and (ii) large wavevectors (high-k). The large anisotropy leads to directional wave propagation in the bulk and on the surface of HMs, and consequently directional emission of light by light sources including quantum emitters [29,30]. Large wavevectors of lightwaves (high-k waves) result in large local density of states, leading to enhanced light-matter interactions (increase in the Purcell factor). This leads to high sensitivity of sensors. Large wavevectors also enable high localization of fields at the nanoscale. Field localization is of great interest in such optical applications as waveguiding [31], sensing [13,20,23] and sub-diffraction imaging [32].

In this review article we cover recent progress in optics due to employment of hyperbolic 
materials. We start in Section 2 with the definition of hyperbolic materials and their types. Brief description of their basic properties to support bulk plasmon polaritions and hyperbolic surface waves are also given in Section 2. In Section 3 we present natural HM materials and artificial material platforms that possess the hyperbolic dispersion, including hyperbolic metamaterials and metasurfaces. In Section 4, we specify the element base provided by HMs. Discussion on HMs applications is provided in Section 5. In this review, we leave quantum nanophotonics [33] and photoluminescence enhancement [6] on HMs to other review articles so an interested reader may find information elsewhere. Finally, Section 5 concludes the paper.

\section{Fundamental}

\subsection{Hyperbolic materials}

Typically, HMs possess uniaxial anistropy with a relative permittivity tensor $[\varepsilon]$

$$
[\varepsilon]=\left(\begin{array}{ccc}
\varepsilon_{o} & 0 & 0 \\
0 & \varepsilon_{o} & 0 \\
0 & 0 & \varepsilon_{e}
\end{array}\right) .
$$

Depending on the sign of $\varepsilon_{o}$ and $\varepsilon_{e}$, the hyperbolic material can be categorized as [4-6]

1. type I: $\varepsilon_{o}>0$ and $\varepsilon_{e}<0$,

2. type II: $\varepsilon_{o}<0$ and $\varepsilon_{e}>0$.

In the case $\varepsilon_{o}>0$ and $\varepsilon_{e}<0$ (type I HM), dispersion band forms a two-fold hyperboloid, see Fig 1. Such scenario can be realized with a metallic wire medium. In the opposite case with $\varepsilon_{o}<0$ and $\varepsilon_{e}>0$ (type II HM) dispersion band has one-fold symmetry. And this scenario can be realized by metal-dielectric multilayer structures.

The dispersion relation of a uniaxial medium is set by equation

$$
\frac{k_{x}^{2}+k_{y}^{2}}{\varepsilon_{o}}+\frac{k_{z}^{2}}{\varepsilon_{e}}=\frac{\omega^{2}}{c^{2}}
$$

For most of naturally occurring materials, the ordinary $\left(\varepsilon_{o}\right)$ and extraordinary $\left(\varepsilon_{e}\right)$ permittivity components are positive, resulting in either spherical isofrequency surfaces for equal permittivities ( $\varepsilon_{o}=\varepsilon_{o}$, isotropic materials) or elliptical isofrequency surfaces when $\varepsilon_{o} \neq \varepsilon_{e}$. When one of the permittivity components becomes negative, equation (2) describes a hyperboloid. Such isofrequency contour supports propagation of light with abnormally large wavevectors (high- $\boldsymbol{k}$ waves), as shown in Fig. 1. On the other hand, along certain directions, there are no any wavevectors available, so light is not allowed to propagate.

\subsection{Bulk plasmon polaritons}

Bulk plasmon polaritons (BPPs) are optical modes propagating (and strongly confined) inside of HMs as illustrated in Fig. 2(a). BPPs are supported by both metallic nanowire [27] and multilayer [13,34-37] HMM structures, as well as natural HMs [1-3]. In the case of metaldielectric multilayer structures, BPPs originates from the hybridization of short-ranged surface plasmon polaritons supported in each thin metal film [37,38]. High wavevectors of BPPs facilitate their tight localization within HMs and poor coupling with light waves. Therefore hing-index prisms or gratings are required in the BPP coupling schemes. The first observation of BPP was realized for a $\mathrm{Au} / \mathrm{SiO}_{2}$ multilayer structure with a Si prism coupler in the near-IR range [34]. High-k waves are also observed by Ge prism for mid-infrared wavelengths [16]. BPPs can be tailored by controlling the geometry of the metamaterial. Their properties can be utilized in 


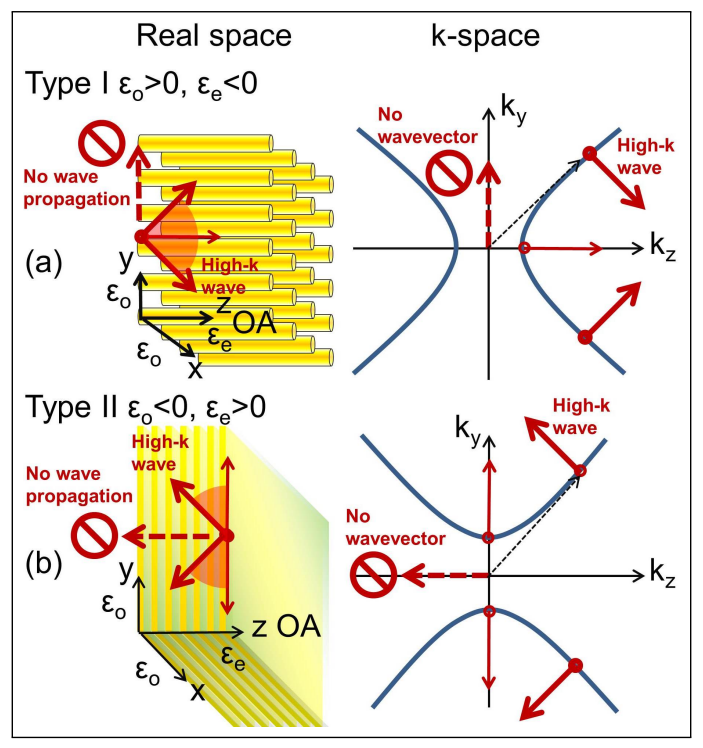

Fig. 1. Illustration of the basic building block of HMM structures. (a) Metallic nanowire structures (type I HM with $\varepsilon_{o}>0$ and $\varepsilon_{e}<0$ ) and (b) Metal-dielectric multilayers (type I HM with $\varepsilon_{o}<0$ and $\varepsilon_{e}>0$ ), and their characteristic dispersion in wavevector space (k-space). The optical axis (OA) is along z-axis.

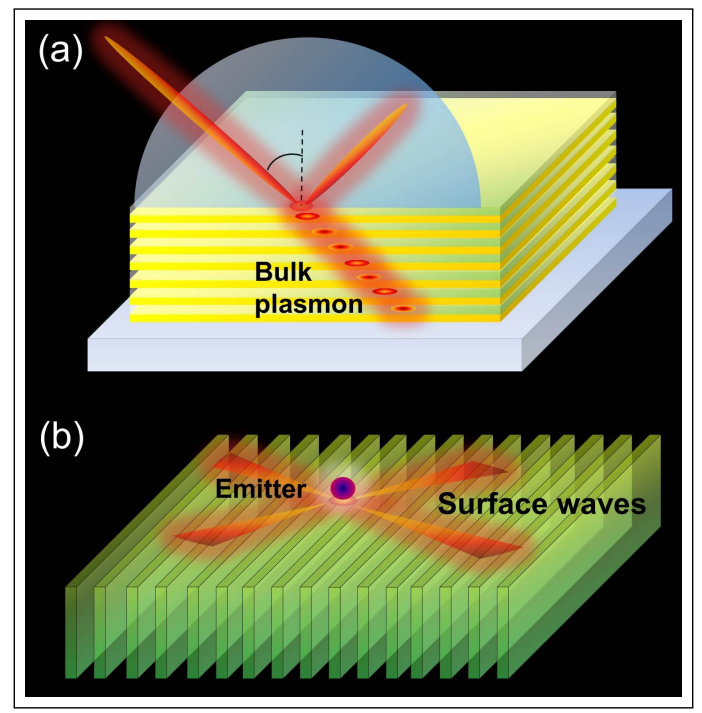

Fig. 2. Illustration of (a) bulk plasmon polariton (BPP) and (b) surface waves on metaldielectric multilayer and trench HMM (type II, $\varepsilon_{o}<0$ and $\varepsilon_{e}>0$ ), respectively. The directional surface waves can be excited by an emitter on trench HMM. 
applications requiring tight localization and directional propagation of high-k waves, for example in sensing [13].

\subsection{Surface waves}

Photonic surface waves (SWs) are optical modes propagating (and strongly confined) at an interface of two dissimilar media including metamaterials $[39,40]$. Amplitudes of SWs exponentially decay away from the boundary. SWs are also termed as surface states or surface modes. Classification of SWs is rather extended, depending on materials and geometry $[39,40]$. Talking about HMs there exist examples of surface waves, which are confined to the HMs interfaces, as illustrated in Fig. 2(b). Such SWs exhibit hyperbolic dispersion in the wavevector space. Surface waves on HMs are sometimes referred to as hyperbolic plasmons [41] or Dyakonov plasmons (DPs) [19,21,29] named after Dyakonov surface waves on interfaces of anisotropic dielectric materials [42-47]. DPs are supported at the interfaces between dielectrics and type II HMMs with its optical axis in the plane of the interface, and have the following features:

1. DPs are directional SWs due to hyperbolic band dispersion.

2. DPs are highly localized at the interface as high- $\mathrm{k}$ waves.

3. DPs are highly sensitive to the change of permittivities in both contacting media.

4. DPs are hybrid-polarized waves with both inseparable TE and TM fields components in contrary to a pure TM-polarized surface plasmon polaritons.

In order to realize a type II hyperbolic HMMs having the optical axis in the plane of the interface shallow metal gratings [17], and metal-dielectric vertical trench structures were proposed and analyzed [19,21, 29, 30, 45, 48,49]. For a fixed wavelength the degree of localization can be controlled by exciting different wavevectors from hyperbolic dispersion band. Dyakonov plasmons on HMMs can be steered by wavelengths [17] or by polarization state [50]. Emitted light from a quantum emitter on the HMM surface can be efficiently coupled to the directional surface waves [29,30] as shown in Fig. 2(b). This suggests the possibility to realize directional quantum emitters placing them on the surface of the HMM that supports directional SWs.

\section{Material platforms}

\subsection{Natural hyperbolic materials}

There are numerous natural materials that exhibit hyperbolic dispersion from ultraviolet (UV) to mid-infrared wavelengths [1-3], for instance, graphite in the UV region, magnesium diboride $\left(\mathrm{MgB}_{2}\right)$ for visible wavelengths. Furthermore, hexagonal boron nitride (h-BN) exhibits hyperbolic dispersion for certain wavelength range in the mid-infrared region due to phonon resonances [51,52]. h-BN becomes a type I HM for $11.76-13.15 \mu \mathrm{m}\left(825-760 \mathrm{~cm}^{-1}\right)$ and type II HM for $6.17-7.35 \mu \mathrm{m}\left(1620-1360 \mathrm{~cm}^{-1}\right)$ [52], respectively. It supports bulk phonon-polaritons, that is the phonon equivalent of BPPs in these wavelength regions. Usually, the optical axis of a h-BN flake is perpendicular to the surface, and as such surface waves are not allowed on the flake. However, when the h-BN layer is patterned in grating geometry, it supports directional surface waves with hyperbolic dispersion. It was demonstrated that directional surface waves propagate on $20 \mathrm{~nm}$ thick h-BN patterned with a nano-grating. The waves were detected around $7.0 \mu \mathrm{m}$ $\left(1425 \mathrm{~cm}^{-1}\right.$ ) by scattering type scanning near-field optical microscopy (s-SNOM) for mid-IR [49]. Directional surface waves have also been observed by s-SNOM in $\alpha$-phase molybdenum trioxide $\left(\alpha-\mathrm{MoO}_{3}\right)$ at $11.2 \mu \mathrm{m}\left(893 \mathrm{~cm}^{-1}\right)$ [53]

While there is a wide variety of natural HMs, hyperbolic dispersion bandwidths are fixed to certain unique intervals. Greater flexibility in acquiring hyperbolic dispersion and relevant 
properties by demand are associated with artificially designed structures, i.e. hyperbolic metamaterials. Therefore, HMMs have been intensively studied in the last decade [5,7]. Mainly, two structural types of HMMs have been realized and studied as building blocks of HMMs : a stack of metal films separated from each other by dielectric spacers-dielectric multilayers [10] and a bundle of metal nanowires in a dielectric host [7,23].

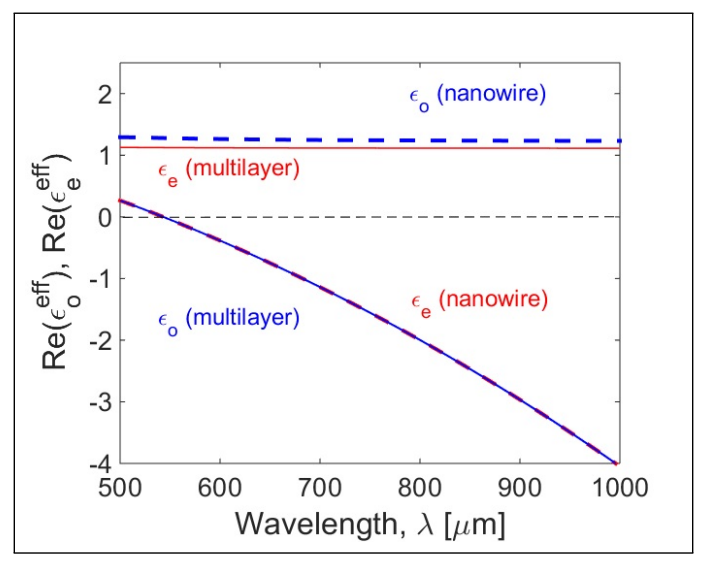

Fig. 3. Effective permittivities of multilayer and nanowire HMM structures composed of gold layers and nanowires calculated by the effective media approximation. The dielectric media is assumed to be air $\left(\varepsilon_{d}=1\right)$. For both structures, the volume fraction of metal is $f_{m}=0.1$. In the visible wavelength interval, the nanowire and multilayer structure behaves as type I $\left(\varepsilon_{o}^{e f f}>0\right.$ and $\left.\varepsilon_{e}^{e f f}<0\right)$ and type II $\left(\varepsilon_{o}^{e f f}<0\right.$ and $\left.\varepsilon_{e}^{e f f}>0\right)$ HMM, respectively.

\subsection{Multilayer structures}

Multilayer structures whose layer thicknesses are sufficiently smaller than that of the wavelength of light can be characterized by effective parameters, i.e. effective permittivities. Based on the effective medium approximation (EMA) $[7,54,55]$, expressions for the effective ordinary and extraordinary permittivities, denoted as $\varepsilon_{o}^{\text {eff }}$ and $\varepsilon_{e}^{\text {eff }}$, respectively, take the form of

$$
\begin{aligned}
& \varepsilon_{o}^{e f f}=f_{m} \cdot \varepsilon_{m}+f_{d} \cdot \varepsilon_{d}, \\
& \varepsilon_{e}^{e f f}=\frac{\varepsilon_{m} \cdot \varepsilon_{d}}{f_{d} \cdot \varepsilon_{m}+f_{m} \cdot \varepsilon_{d}},
\end{aligned}
$$

where $\varepsilon_{m}, \varepsilon_{d}$ and $f_{m}, f_{d}$ are the permittivities of metal and dielectric layers, and fractions of metal and dielectric in the multilayer, respectively. Eq. (3) and (4) suggest that the effective permittivities can be widely tuned by combination of different metals $\left(\varepsilon_{m}\right)$ and dielectrics $\left(\varepsilon_{d}\right)$, as well as by adjusting the volume fraction of each material, $f_{m}$ and $f_{d}$. In this way it is possible to modify (in some extent) effective optical properties accordingly to the requirements. Note that EMA does not always hold: there are many constrains on the thicknesses of the layers, number of periods, and range of angles of incidence $[16,56,57]$. Moreover, for very large values of wavevectors, dispersion starts to deviate significantly from that predicted by the effective medium approximation [58].

The relative easiness of fabrication and accessible planar nanotechnology of deposition of alternating layers of metal and dielectric, multilayer HMMs have been extensively studied with various combination of materials. $\mathrm{Au} / \mathrm{SiO}_{2}$ multilayers were fabricated for the observation of high$\mathrm{k}$ BPPs excited with the help of a Si prism [34]. Apart from Au as a material for conductive films for visible to near-IR wavelengths [16,34], other materials like silver $(\mathrm{Ag})$ [11,59-61], titanium 
nitride (TiN) [62], aluminum-doped zinc oxide (AZO) [63], and doped semiconductors [10] have been reported plasmonic constituent layers of HMMs. In case of semiconductors, multilayer HMM can be made of the same semiconductor but with different doping levels: highly doped layers serve as metal and low doped layers serve as dielectric [15]. Apart from these structures, there are other variations of metal-dielectric multilayer HMMs [64], such as, trench and high aspect ration plasmonic grating structures as a vertical version of multilayer HMM [19-21], tubular HMMs [64], fishnet structures that function as Magnetic HMM [65].

\subsection{Nanowire structures}

Similarly to the multilayers, in the case of metal nanowire structures in a dielectric host, the effective ordinary and extraordinary permittivities can be expressed by [7]:

$$
\begin{gathered}
\varepsilon_{o}^{e f f}=\frac{\left(1+f_{m}\right) \varepsilon_{m} \cdot \varepsilon_{d}+\left(1-f_{m}\right) \cdot \varepsilon_{d}^{2}}{\left(1+f_{m}\right) \varepsilon_{d}+\left(1-f_{m}\right) \cdot \varepsilon_{m}}, \\
\varepsilon_{e}^{e f f}=f_{m} \cdot \varepsilon_{m}+f_{d} \cdot \varepsilon_{d},
\end{gathered}
$$

where $\varepsilon_{m}$ and $\varepsilon_{d}$ are the permittivities of metallic nanowire and dielectric host, and $f_{m}$ and $f_{d}$ are corresponding volume fractions of metal nanowire and dielectric matrix. Typically, nanowirebased HMMs are fabricated by Au nanowires grown in porous alumina template [22, 26, 27]. However, there are some nanowire structures based on transparent conductive oxides (TCO), such as, indium-tin-oxide (ITO) [28] and aluminum-doped zinc oxide (AZO) [25]. Their zero crossing wavelengths for one of the effective permittivities can be tuned in the near-infrared and mid-infrared ranges, depending on the periodicity and doping level.

As an example of HMMs' effective permittivities made of metal-dielectric multilayers and metallic nanowires, we plot both effective permittivities, $\varepsilon_{o}^{\text {eff }}$ and $\varepsilon_{e}^{\text {eff }}$, in the visible and near-IR range as shown in Fig. 3. Note that as metal we use gold whose permittivity, $\varepsilon_{m}$, is characterized by the Drude-Lorentz model and as dielectric we use $\mathrm{SiO}_{2}$. Both HMM structures have a zero crossing wavelength around $\lambda=500 \mathrm{~nm}$, but for different effective components: $\varepsilon_{o}^{\text {eff }}$ for the multilayer and $\varepsilon_{e}^{e f f}$ for the nanowires structure, respectively. For longer wavelengths, the metamaterials behave as either type I HMMs $\left(\varepsilon_{o}^{e f f}<0\right.$ and $\left.\varepsilon_{e}^{\text {eff }}>0\right)$ in case of nanowires or type II HMMs ( $\varepsilon_{o}^{e f f}<0$ and $\left.\varepsilon_{e}^{e f f}>0\right)$ in case of the stack.

\section{Advanced structures}

\subsection{Metasurfaces}

Metasurfaces (MSs), whose thicknesses are significantly smaller than operating wavelength are thin film (or two-dimensional) equivalent of metamaterials. There are several designs of metasurfaces that support surface waves (or eigen modes) with hyperbolic dispersion. The simplest design is a one-dimensional subwavelength grating of plasmonic materials, such as, for example, graphene strips for $\mathrm{THz}$ frequencies [41], or a silver grating for visible wavelengths [17], as shown in Fig. 4(a). While one-dimensional gratings possess required in-plane anisotropy by default, two dimensional gratings anisotropy must be provided by structural design to ensure supporting of hyperbolic waves. An example of such in-plane anisotropy is given in Ref. [66,67], where a square lattice of gold elliptic particles support both TE- and TM-polarized surface waves with hyperbolic dispersion at the near-infrared wavelengths. On the microwave frequencies, the hyperbolic surface waves were theoretically analyzed and observed for various metasurfaces [68,69] as shown in Fig. 4(c) and (d). In these cases, the frequency dependence of metasurfaces' dispersion results in possible propagation direction steering by frequency. Moreover, HMMs made of electronic components, such as capacitors and inductors, provide hyperbolic dispersion, and one can steer directional surface waves by input polarization states [50]. 


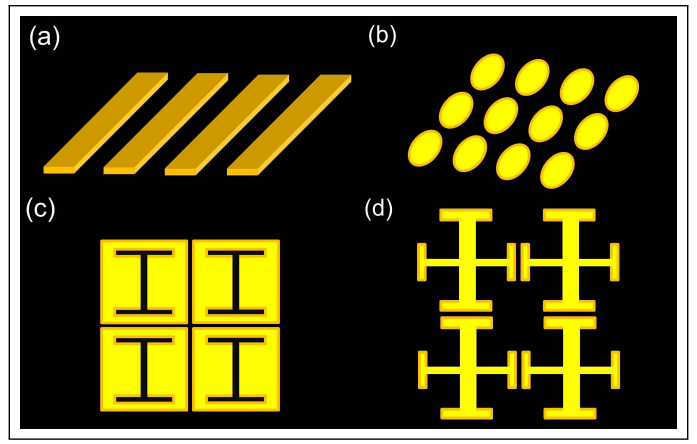

Fig. 4. Examples of hyperbolic metasurfaces of subwavelength (a) graphene [41] and Ag gratings [17]. (b) Elliptic Au particles [66,67]. (c),(d) Diffrent designs of copper (Cu)-based metasurfaces for microwave frequencies $[68,69]$.

\subsection{Hypercrystals}

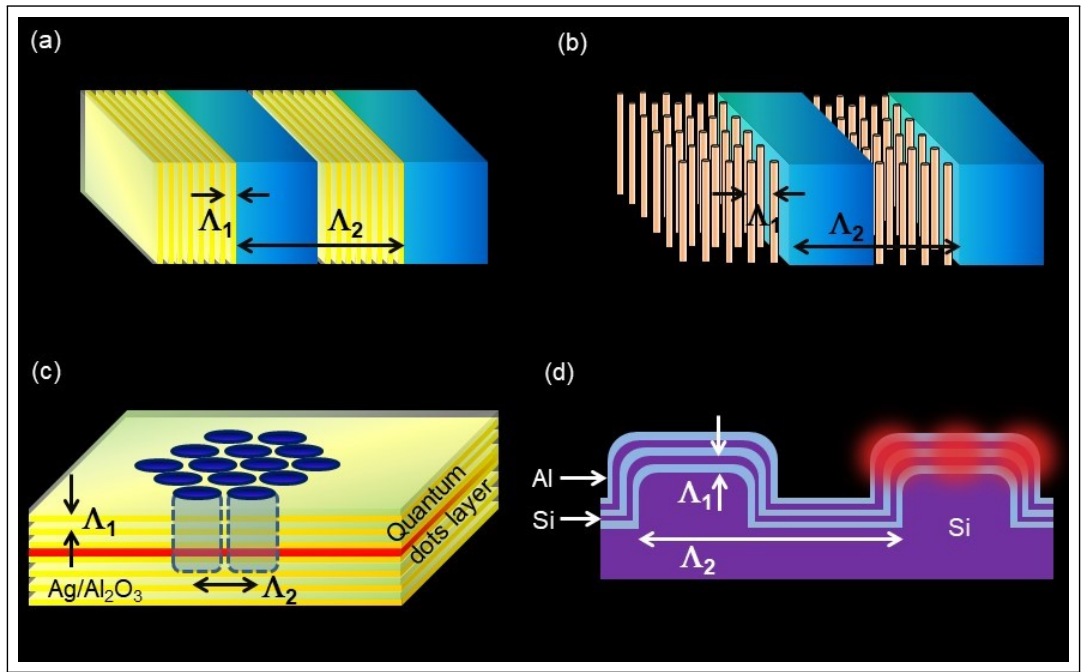

Fig. 5. Hypercrystal structures. (a) Metal-dielectric multilayer structures with two different periods [35,70]. (b) Cobalt nanowire-based structures with two different periods [71]. (c) $\mathrm{Ag} / \mathrm{Al}_{2} \mathrm{O}_{3}$ multilayer $\mathrm{HMM}$ with grating and quantum dots layer embedded [72]. (d) Cross-section of a trench structure covered with Al/Si multilayer HMM [73, 74]. The hypercrystals consist of small $\Lambda_{1}$ and large periods, $\Lambda_{2}$, respectively. Note that (a) has $\Lambda_{1}$ and $\Lambda_{2}$ along the same axis while (b) and (c) have $\Lambda_{1}$ and $\Lambda_{2}$ along different axes.

A hypercrystal [70] or multi-scale hyperbolic metamaterial [35] is a hyperbolic metamaterials with at least two different periods or features size scaling. The initially proposed design of a hypercrystal represents a nested periodic structure, where one or both structural elements of the unitary cell are in turn composed of metal-dielectric multilayer stacks with much smaller period as illustrated in Fig. 5(a). The first experimental realization of hypercrystal was done in different topology as shown in Fig. 5(b). Instead of metal-dielectric multilayer with smaller period, the hypercrystal consists of cobalt nanowires with subwavelength period $(\approx 20 \mathrm{~nm})$ and isotropic layer arranged in larger period $(\approx 2 \mu \mathrm{m})$ [71]. The cobalt nanowires are aligned by application of external magnetic field to a cobalt nanoparticles. The structure functions as a 
hypercrystal for mid-infrared wavelengths over $3 \mu \mathrm{m}$ in wavelength. Since then, there have been a few experimental realization of hypercrystals. As shown in Fig. 5(c), the hypercrystal is made of a multilayer HMM with a deeply subwavelength period, while a two-dimensional hexagonal array of protruding holes is arranged with a larger period comparable with the wavelength of light. Additionally multiple quantum dots are embedded in the multilayer HMM. When quantum dots are excited, the emitted light is efficiently out-coupled by the grating [72]. In this work, the fine HMM works for the enhancement of the spontaneous emission rate while broadband efficient out-coupling of emitted light is performed due to the grating with larger period.

A Si grating structure coated with a multilayer HMM has been realized [73,74] as illustrated in Fig. 5(d). Here the grating with a larger period allows excitation of various high-k bulk plasmon modes in the multilayer HMM acting as a grating coupler. In this manner, we do not need bulky coupling elements, such as, prisms. Besides, the highest possible wavevector provided by the prism coupling scheme is limited by the refractive index of prism material, which can be at the best only $n=4$ for Ge prisms. Therefore, grating couplers combined with a HMM in the hypercrystal fashion are potentially very interesting objects for various on-chip applications.

\subsection{Waveguide}

Waveguides employing HMs hold a potential to confine guided modes within a lot smaller space than conventional dielectric waveguide structures thanks to support of high-k BPP modes. In dielectric waveguides, an effective mode index is similar to the refractive index of the dielectric core and cladding layers, setting a limit to the maximum value of the effective mode index. A high-k propagating wave typically has a large effective mode index and consequently short effective wavelength. For example, in the case of a waveguide with the multilayer HMM core sandwiched by dielectric cladding layers [Fig. 6(a)], BPPs are tightly confined in the core with the very short penetration depth or tale of evanescent field in the cladding [34]. Similarly, a HMM waveguide with the gold nanowire core has been experimentally demonstrated with the effective mode index of up to 10 [27]. Apart from the HMM core structures, waveguide structures with the HMM cladding have been theoretically studied in two configurations: Lateral multilayer HMM [Fig. 6(b)] [31,75] and vertical multilayer HMM [Fig. 6(c)]. As shown in [76] such waveguides support slow light modes with group velocity up to 10 times slower than speed of light in vacuum.

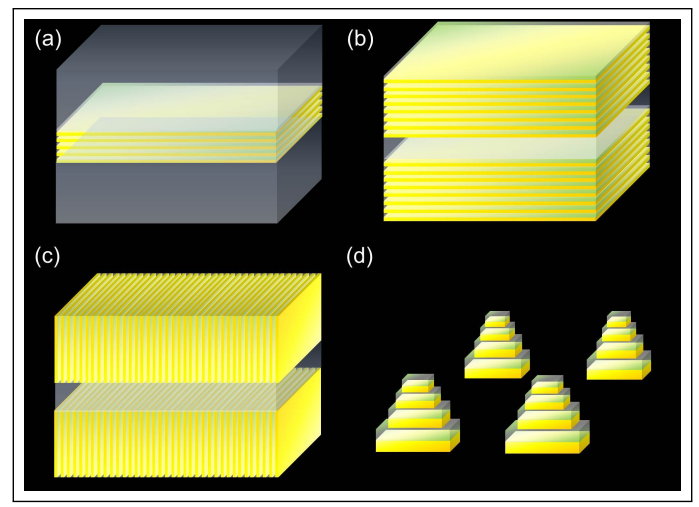

Fig. 6. HMM waveguide structures made of (a) HMM core [16,31,37]. (b) Multilayer HMM cladding [31,75]. (c) Vertical multilayer HMM cladding [76]. (d) Optical cavities made of two to four periods of $\mathrm{Ag} / \mathrm{Ge}$ multilayer HMMs [60]. 


\subsection{Optical cavity}

An optical cavity traps light within the limited volume by reflecting light back and force between the boundaries. Cavities are the backbone in numerous applications in lasing, nonlinear optics, and sensing. Conventionally, the resonant wavelength of a cavity depends strongly on the sizes and material parameters (refractive index) of the cavity. As the effective wavelength is inversely proportional to the refractive index, to make the cavity small a material with a high refractive index is required. However, due to the limitation of refractive indices available from natural materials, the minimum sizes of the optical cavities are of the order of a wavelength.

Optical cavities made of multilayer HMMs patterned in a pyramid shape as illustrated in Fig. 6(d) were demonstrated [60]. The HMM was made of two to four pairs of Ag/Ge with $20 \mathrm{~nm}$ and $30 \mathrm{~nm}$ thickness, respectively. Due to the large wavevector or effective mode index in the HMM (as large as 17), the size of the cavity is a few hundred of nanometers as opposed to $1.5 \mu \mathrm{m}$ wavelength, which is approximately one order of magnitude smaller than what can be achieved by natural materials. Optical cavities made of ribbons of natural $\mathrm{HM}, \mathrm{h}-\mathrm{BN}$, were also realized for mid-infrared sensing applications [77].

\section{Applications}

\subsection{Negative refraction}

Initially, negative refraction of electromagnetic waves was predicted [78] and demonstrated [79] on left-handed materials (LHMs) or double negative materials (DNMs) whose permittivity and permeability become simultaneously negative $(\varepsilon<0$ and $\mu<0)$ on microwave frequencies. The LHMs were assembled of metallic wires and split rings resonators. Negative refraction leads to unique applications, such as subwavelength imaging and planar lens designs [80].

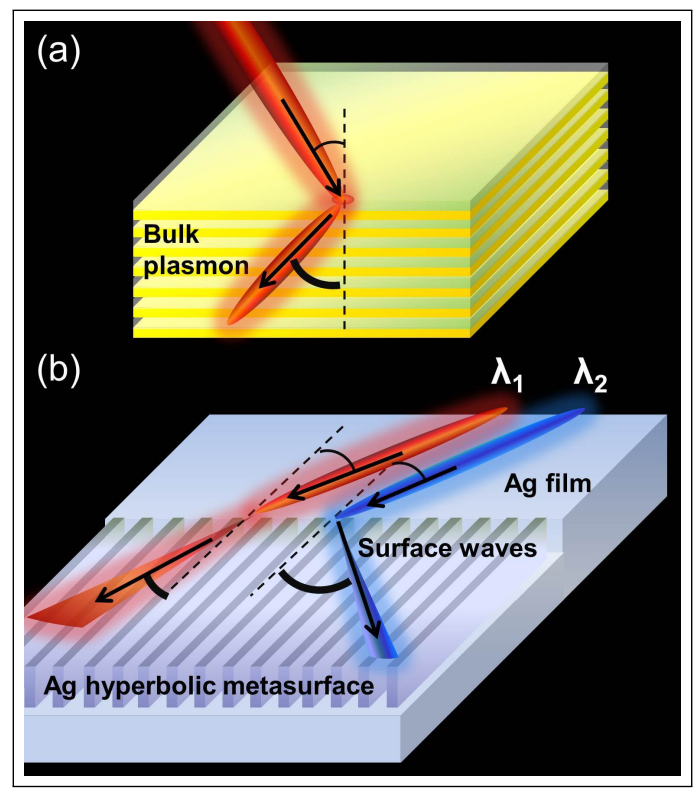

Fig. 7. Negative refraction of (a) bulk plasmon polariton propagating inside multilayer of doped semiconductor [10] and (b) surface waves on plane boundary of Ag hyperbolic metasurface [17]. Note that the surface wave with $\lambda_{1}$ experiences positive refraction while $\lambda_{2}$ goes under negative refraction.

Quite painful requirement for the negative permeability can be withdrawn if HMMs are 
involved. Negative refraction in a multilayer HMM was realized in the mid-infrared wavelength region of around $10 \mu \mathrm{m}$ where the multilayer structure is of type I HMM $\left(\varepsilon_{o}^{\text {eff }}>0, \varepsilon_{e}^{\text {eff }}<0\right)$ as illustrated in Fig. 7(a) [10]. The structure was composed of alternating layers of highly doped InGaAs (plasmonic) and intrinsic AlInAs (dielectric) layers. The wavelength, where negative refraction takes place, can be tuned by the doping level of the semiconductor layers. Later on, negative refraction was observed for $\mathrm{Ag}$ nanowires in an anodic porous alumina matrix that behaves as type I HMM for visible wavelengths [81].

Negative refraction of directional surface waves was also demonstrated at visible wavelengths for a Ag hyperbolic metasurface, which is nothing else but a subwavelength one-dimensional grating [17]. As illustrated in Fig. 7(b), negative refraction takes place at certain wavelengths while for other wavelengths conventional positive refraction occurs due to the material dispersion. This wavelength dependent negative refraction can be used for the steering of optical signals with different wavelengths.

\subsection{Hyperlens - super resolution microscopy}

A subwavelength optical imaging device made of HMM, hyperlens [82], takes advantage of high wavevectors of the BPP modes. The BPPs transfer full near-field information from a point source (scatterer or emitter) at one side of the HMM to the other side as illustrated in Fig. 8. When a scattering object (or point source) is placed near the inner curvature of HMM and is illuminated, light scattered by the object in the near-field zone has both high-k evanescent components and low-k propagating components. The high-k evanescent field can be coupled to BPP modes in the HMM of curved geometry, and thus propagate toward the outward side of the hyperlens. There by out-coupling of BPPs to far-field via conventional lens it is possible to obtain a super-resolution image beyond the diffraction limit [32]. The resolution and magnification of the image are dependent on the ratio between the inner and outer radii of the hyperlens. Note that this hyperlens operates in type I HM regime $\left(\varepsilon_{o}^{e f f}>0\right.$ and $\left.\varepsilon_{e}^{e f f}<0\right)$, so that the BPP modes with flat hyperbolic dispersion propagate perpendicular to the multilayers (radially).

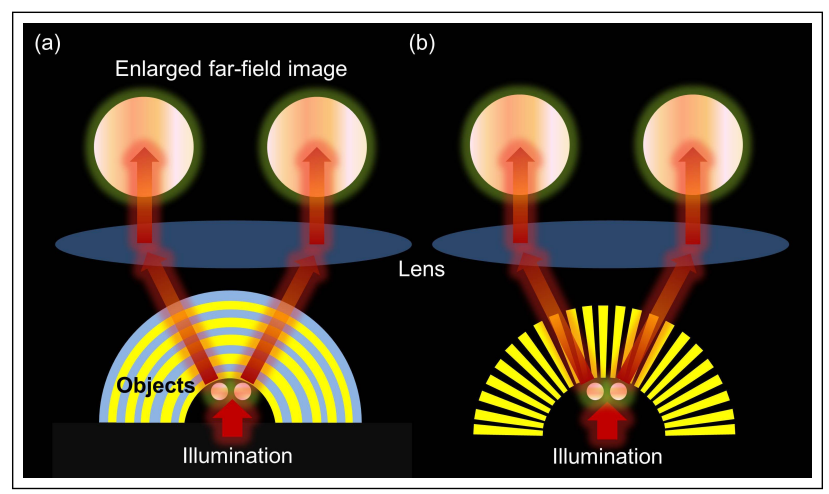

Fig. 8. Hyperlens made of (a) mutlilayer HMM in a hemi-cylindrical [83] and spherical [85, 86] geometry, and (b) plasmonic nanowires HMM in a radial geometry [87]. Subdiffraction objects are resolved by hyperlens in the far-field image.

The initial demonstration of hyperlens was realized by a cyrindrical curved alternating multilayer of $\mathrm{Al}_{2} \mathrm{O}_{3}$ and $\mathrm{Ag}$ with 8 periods [83]. It operates on the UV wavelength of $365 \mathrm{~nm}$ and achieves the subwavelength resolution of $130 \mathrm{~nm}$. At the same time, the surface wave versions of hyperlens made of rings of poly(methyl methacrylate) (PMMA) deposited on a gold film was reported [84], achieving the resolution of $70 \mathrm{~nm}$ for the visible wavelength of $495 \mathrm{~nm}$. The superlensing effect was given by the alternating layer of gold-air and gold-PMMA interfaces, 
which functions as hyperbolic metamaterials [29]. Later, the hyperlens in a semi-spherical curved multilayer HMM composed of 9 periods of $\mathrm{Ag} / \mathrm{Ti}_{3} \mathrm{O}_{5}$ was demonstrated for visible wavelength of $410 \mathrm{~nm}$ [85]. Reversely, the hyperlens is suggested to achieve high resolution patterning in photolithography [86]. Apart from the multilayer geometry, nanowires arranged in radial geometry on a planar surface was demonstrated in the microwave frequency [88] and visible wavelengths [87]. As extension of such configuration a hyperlens with graphene ribbons in radial geometry was proposed to focus THz radiation in planar geometry [89] as shown in Fig. 8(b).

Generally, in the case of hyperlens made of type I HMM, the excitation beam is also transmitted through the HMM, creating background noise in the image plane and thus screening the image signal. For weak scattering objects such background noise might be a serious issue. In order to eliminate the background illumination, dark field hyperlens that adopts type II HMM $\left(\varepsilon_{o}^{e f f}<0\right.$ and $\varepsilon_{e}^{e f f}>0$ ) was proposed [90,91]. The dark field hyperlenses are also made of metal-dielectric multilayers operating in type II regime, as illustrated in Fig. 8(a). Only light scattered from the object with large wavevectors is coupled to high-k BPP modes that propagate in the hyperlens, while other propagating waves, such as, illumination light with small wavevectors, are blocked and reflected by the HMM. In this manner, strong background can be almost completely eliminated in the image. Additionally a hyperlens composed of magnetic materials was proposed as an alternative to the dark-field hyperlens. [92].

\subsection{Photonic spin Hall effect}

The photonic spin Hall effect (PSHE) [93] or spin Hall effect of light (SHEL) [94] is a polarizationdependent transverse beam shifting upon transmission or reflection from an interface [95-99]. It is originally known as the transverse Imbert-Federov beam shift [100]. When incident light with either right-hand or left-hand circular polarization is transmitted or reflected at an interface of materials, subsequent light beam is shifted in the transverse plane depending on the polarization as illustrated in Fig. 9(a).

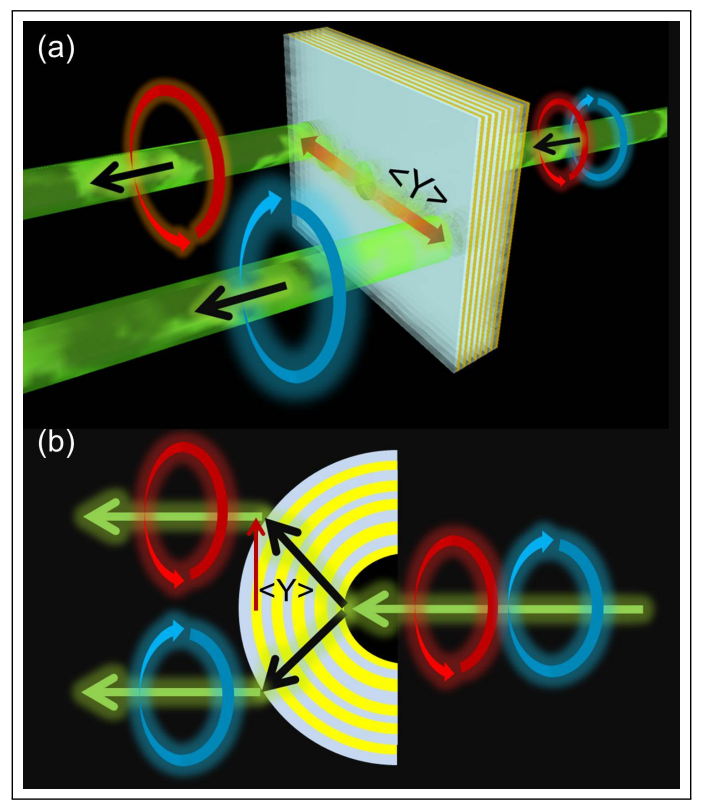

Fig. 9. Conceptual illustration of photonic spin Hall effect through (a) a planar [14] and (b) hemi-cyrindrical multilayer hyperbolic metamaterial (hyperlens) [101]. The transverse beam shift along the $\mathrm{y}$-axis is denoted as $\langle Y\rangle$. 
In contrast to isotropic media, photonic spin Hall effect on anisotropic media depends on anisotropy in a straightforward manner [102]. This enables to enhance and tune the transverse beam shift by anisotropy. So HMMa as materials with extreme anisotropy can be employed to facilitate the PSHE. The spin Hall effect in HMMs has been theoretically [101,103-105] and experimentally $[14,50]$ studied recently. First experimental observation of the spin Hall effect in HMMs was reported for microwave frequencies, where directional surface waves on HMM were steered by input polarization states [50]. Recently the PSHE in transmission through a multilayer $\mathrm{HMM}$ composed of alternating layers of $\mathrm{Au}$ and $\mathrm{Al}_{2} \mathrm{O}_{3}$ has been experimentally demonstrated for visible wavelengths. The transverse beam shift is very sensitive to the incident angle on the order of $\approx 0.05^{\circ}$ due to the extremely large anisotropy of the HMM. Compared with natural birefringent media, the transverse beam shift in HMMs is two orders of magnitude more sensitive to the angle of incidence change. The shift was achieved with a lot thinner structure of a few hundreds of nanometers as opposed to dosens of microns of natural anisotropic materials.

Moreover, photonic spin Hall effect in hyperlens with circular geometry of a multilayer HMM, was theoretically studied as shown in Fig. 9(b). In such curved HMM geometry, the splitting of beams for different circular polarizations is enhanced [101]. Photonic spin-Hall effect in HMM enables to manipulate light at nanoscale by means of spin, incident angle, and wavelength, which may lead to potential applications for spin-dependent switching, beam splitters, filters, optical diodes [93], surface sensors [106, 107], and quantum information processing [93,94].

\subsection{Refractive index sensing}

HMMs can be used in sensing applications. Enhanced density of optical states enables high sensitivity toward the change of refractive index caused by the presence of analyte. Typically the presence and concentration of analyte is detected as a frequency shift of optical modes whose wavevector is sensitive to the change of refractive index. Highly localized bulk and surface modes on HMMs can be used as probing tools for analytes. Conventionally, plasmonic sensors are based on surface plasmon polaritons (SPPs) [108] and adopt a prism coupler (Kretchmann configuration) or grating coupler on a metal film in order to match wavevector between the surface waves and external light. Both prism and grating-based plasmonic sensors measure the small changes in refractive index in the vicinity of the metal/dielectric interface in terms of the plasmon resonant shift.

Recently, it has been reported that BPPs in both metal nanowire [23] and planar multilayer HMM [13] structures exhibit extremely high sensitivity in biosensing applications. Both structures achieved bulk refractive index sensitivity of over $30,000 \mathrm{~nm} / \mathrm{RIU}$ (per refractive index unit). Gold nanowire array HMMs were proposed for biosensors for near-infrared wavelengths [23]. The nanowire HMM is type I HMM. It supports a guided mode (bulk plasmon polariton) in a nanorod array, where analyte resides as illustrated in Fig. 10(a). In this work, a prism coupler was placed on top of the $500 \mathrm{~nm}$ thick nanowire HMM layers in order to excite the BPP mode that senses the change of refractive index caused by the concentration variation of the analyte.

Another HMM biosensing platform is based on a metal-dielectric multilayer that demonstrates high sensitivity for cancer biomarkers in broadband range from visible to near-IR wavelengths [13]. The sensing unit consists of a multilayer HMM with a 2D Au diffraction grating placed on the HMM in contact with the analyte liquid as shown in Fig. 10(b). The multilayer HMM supports higher order BPP modes each of which has different sensitivity toward the change of refractive index of the analyte. The concentration difference is detected via the resonance shift of the reflection peak in terms of the wavelength. With the grating coupler, the excitation of high-k modes is possible without the need of a bulky prism coupler, which is advantageous for compactness and multiplexing of sensing units. 


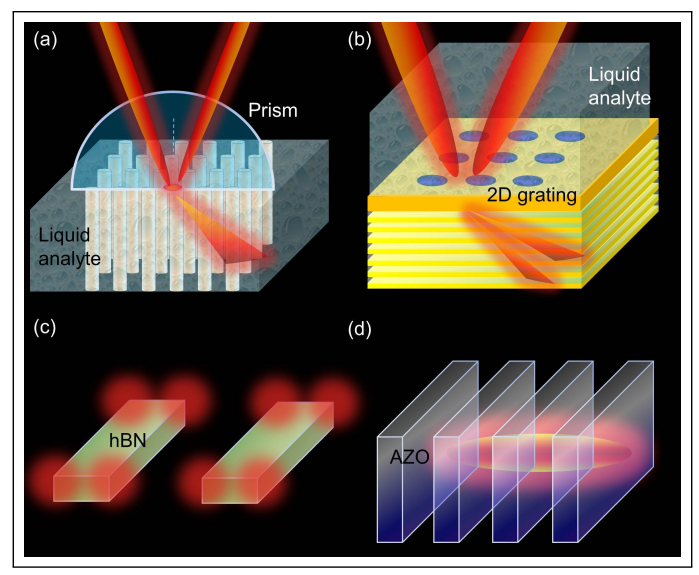

Fig. 10. Sensing by HMs. (a) Au nanowire HMM (type I) with prism coupler [23], and (b) $\mathrm{Au} / \mathrm{Al}_{2} \mathrm{O}_{3}$ multilayer HMM (type II) with 2D grating coupler on Au film [13] for refractive index sensing. (c) h-BN ribbon resonator [77], and (d) Al-doped zinc oxid (AZO) trench HMM (type II) [20] for mid-infrared vibrational spectroscopy. There are bulk plasmon polariton modes propagating in HMM structures in (a),(b), and (d) cases.

\subsection{Mid-infrared absorption sensing}

The mid-infrared (IR) absorption spectroscopy in the wavelength range of $2.5-20 \mu \mathrm{m}$ (4000 $500 \mathrm{~cm}^{-1}$ ) is extensively utilized as a unique label-free sensing scheme to detect molecules thanks to their characteristic absorption of infrared light at certain wavelengths unique to chemical bondings [109]. The wavelength region includes the absorption bands of interesting biologically relevant molecules [110]. However, due to the mismatch of the mid-infrared light wavelength on the order of microns as opposed to nanometer size molecules, the interaction between them is typically weak, resulting in small absorption signatures. To address this issue, surface enhanced infrared absorption spectroscopy (SEIRAS) $[111,112]$ has been extensively developed in last decade by using plasmonic nanostructures that confine mid-IR light at the nanoscale and improve photon-matter interactions.

Recently, it has been shown that light absorption by molecules can be enhanced by placing the molecules on a h-BN ribbon array [Fig. 10(c)] [77]. Each h-BN ribbon functions as a resonator localizing mid-IR light in the range of $6.17-7.35 \mu \mathrm{m}\left(1620-1360 \mathrm{~cm}^{-1}\right)$. When analyte molecules cover the h-BN resonators, light absorption by analyte is significantly enhanced. In this case, phonon-resonance was exploited for molecular vibrational spectroscopy as opposed to conventional SEIRA based on plasmonic nanostructures.

The enhancement of infrared absorption in plasmonic trench structures that function as hyperbolic metamaterials was demonstrated in reference [20]. The HMM is composed of plasmonic trenches of aluminum-doped zinc oxide (AZO), and functions as type II HMM $\left(\varepsilon_{o}^{\text {eff }}<\right.$ 0 and $\left.\varepsilon_{e}^{e f f}>0\right)$ in a broad wavelength range above $3 \mu \mathrm{m}$, covering most of the interesting wavelength region [19]. The trench HMM supports bulk plasmon modes that propagate and interact with analyte inside trenches. Thus, BPPs can be employed contributing to the absorption signature of the molecules. A $5 \mathrm{~nm}$ thick silica layer conformally coated around the AZO trenches absorbs light with wavelengths around $8 \mu \mathrm{m}$. It was used as a model analyte [113]. The enhanced absorption is achieved by the combination of bulk plasmon modes in the trenches and the large surface area of the trench geometry. Such plasmonic nanotrench structures may serve as a highly sensitive biochemical sensing platform for mid-infrared vibrational spectroscopy. 


\section{Conclusion}

In this article, we have reviewed the current progress in hyperbolic materials and optical applications driven by their hyperbolic dispersion. Bulk plasmon polaritons and directional hyperbolic surface waves are responsible for most of intriguing optics with HMs. We focus on characteristics of natural and artificial hyperbolic materials, material platforms that support both types of lightwaves, and various novel applications from waveguiding to super-resolution microscopy. In difference with natural HMs, optical properties of hyperbolic metamaterials can be tailored for different purposes by design parameters and materials involved. The major challenges so far in applications of HMs have been the fabrication of metamaterial structures for the visible and near-infrared wavelength regions. However, steady advancement in nanofabrication technologies and widening the nomenclature of natural HMs can mitigate the challengers.

\section{Funding}

Independent Research Fund Denmark, DFF Research Project 2 (8022-00387B); Villum Fonden, DarkSILD project (11116); Direktør Ib Henriksens Fond, Denmark.

\section{References}

1. J. Sun, N. M. Litchinitser, and J. Zhou, "Indefinite by Nature: From Ultraviolet to Terahertz," ACS Photonics 1, 293-303 (2014).

2. K. Korzeb, M. Gajc, and D. A. Pawlak, "Compendium of natural hyperbolic materials," Opt. Express 23, 25406 (2015).

3. E. E. Narimanov and A. V. Kildishev, "Metamaterials: Naturally hyperbolic," Nat. Photonics 9, 214-216 (2015).

4. Y. Guo, W. Newman, C. L. Cortes, and Z. Jacob, "Applications of hyperbolic metamaterial substrates," Adv. OptoElectronics 2012, 1-9 (2012).

5. A. Poddubny, I. Iorsh, P. Belov, and Y. Kivshar, "Hyperbolic metamaterialsals," Nat. Photonics 7, 948-957 (2013).

6. P. Shekhar, J. Atkinson, and Z. Jacob, "Hyperbolic metamaterialsals: fundamentals and applications," Nano Convergence 1, 14 (2014)

7. L. Ferrari, C. Wu, D. Lepage, X. Zhang, and Z. Liu, "Hyperbolic metamaterialsals and their applications," Prog. Quantum Electron. 40, 1-40 (2015).

8. L. Lu, R. E. Simpson, and S. K. Valiyaveedu, "Active hyperbolic metamaterialsals: progress, materials and design," J. Opt. 20, 103001 (2018).

9. J. S. T. Smalley, F. Vallini, X. Zhang, and Y. Fainman, "Dynamically tunable and active hyperbolic metamaterialsals," Adv. Opt. Photonics 10, 354 (2018).

10. A. J. Hoffman, L. Alekseyev, E. E. Narimanov, C. Gmachl, and D. L. Sivco, "Negative refraction in mid-infrared semiconductor metamaterials," Nat. Mater. 6, 946-950 (2007).

11. H. N. S. Krishnamoorthy, Z. Jacob, E. Narimanov, I. Kretzschmar, and V. M. Menon, "Topological transition in metamaterials," Science 336, 205-209 (2012).

12. S. Ishii, A. V. Kildishev, E. Narimanov, V. M. Shalaev, and V. P. Drachev, "Sub-wavelength interference pattern from volume plasmon polaritons in a hyperbolic medium," Laser \& Photonics Rev. 7, 265-271 (2013).

13. K. V. Sreekanth, Y. Alapan, M. ElKabbash, E. Ilker, M. Hinczewski, U. A. Gurkan, A. De Luca, and G. Strangi, "Extreme sensitivity biosensing platform based on hyperbolic metamaterials," Nat. Mater. 15, 621-627 (2016).

14. O. Takayama, J. Sukham, R. Malureanu, A. V. Lavrinenko, and G. Puentes, "Photonic spin Hall effect in hyperbolic metamaterials at visible wavelengths," Opt. Lett. 43, 4602-4605 (2018).

15. P. Sohr, D. Wei, S. Tomasulo, M. K. Yakes, and S. Law, "Simultaneous large mode index and high quality factor in infrared hyperbolic metamaterials," ACS Photonics 5, 4003-4008 (2018).

16. M. Mahmoodi, S. H. Tavassoli, O. Takayama, J. Sukham, R. Malureanu, and A. V. Lavrinenko, "Existence Conditions of High-k Modes in Finite Hyperbolic Metamaterials,” Laser Photonics Rev. 13, 1800253 (2019).

17. A. A. High, R. C. Devlin, A. Dibos, M. Polking, D. S. Wild, J. Perczel, N. P. De Leon, M. D. Lukin, and H. Park, "Visible-frequency hyperbolic metasurface," Nature 522, 192-196 (2015).

18. J. S. T. Smalley, F. Vallini, S. A. Montoya, L. Ferrai, S. Shahin, C. T. Riley, B. Kanté, E. E. Fullerton, Z. Liu, and Y. Fainman, "Luminescent hyperbolic metasurface," Nat. Commun. 8, 13793 (2017).

19. O. Takayama, E. Shkondin, A. Bogdanov, M. E. Aryaee Panah, K. Golenitskii, P. A. Dmitriev, T. Repän, M. Malreanu, P. Belov, F. Jensen, and A. V. Lavrinenko, "Midinfrared surface waves on a high aspect ratio nanotrench platform," ACS Photonics 4, 2899-2907 (2017).

20. E. Shkondin, T. Repän, M. E. Aryaee Panah, A. V. Lavrinenko, and O. Takayama, "High Aspect RAtio Plasmonic Nanotrench Structures with Large Active Surface Area for Label-Free Mid-Infrared Molecular Absorption Sensing,' ACS Appl. Nano Mater. 1, 1212-1218 (2018). 
21. O. Takayama, P. Dmitriev, E. Shkondin, O. Yermakov, M. E. Shkondin, O. Yermakov, M. E. A. Panah, K. Golenitskii, F. Jensen, A. Bogdanov, and A. V. Lavrinenko, "Experimental Observation of Dyakonov Plasmons," Semiconductors 52, 442-446 (2018).

22. M. A. Noginov, Y. A. Barnakov, G. Zhu, T. Tumkur, H. Li, and E. E. Narimanov, "Bulk photonic metamaterial with hyperbolic dispersion," Appl. Phys. Lett. 94, 151105 (2009).

23. V. Kabashin, P. Evans, S. Pastkovsky, W. Hendren, G. Wurtz, R. Atkinson, R. Pollard, V. Podolskiy, and V. Zayats, "Plasmonic nanodrod metamaterials for biosensing," Nat. Mater. 8, 867-871 (2009).

24. P. Ginzburg, F. J. R. Fortuño, G. A. Wurtz, W. Dickson, A. Murphy, F. Morgan, R. J. Pollard, I. Iorsh, A. Atrashchenko, P. A. Belov, Y. S. Kivshar, A. Nevet, G. Ankonina, M. Orenstein, and A. V. Zayats, "Manipulating polarization of light with ultrathin epsilon-near-zero metamaterials," Opt. Express 21, 14907 (2013).

25. C. T. Riley, J. S. T. Smalley, K. W. Post, D. N. Basov, Y. Fainman, D. Wang, Z. Liu, and D. J. Sirbuly, "High-Quality, Ultraconformal Aluminum-Doped Zinc Oxide Nanoplasmonic and Hyperbolic Metamaterials," Small 12, 892-901 (2015).

26. M. E. Nasir, S. Peruch, N. Vasilantonakis, W. P. Wardley, W. Dickson, G. A. Wurtz, and A. V. Zayats, "Tuning the effective plasma frequency of nanorod metamaterials from visible to telecom wavelengths," Appl. Phys. Lett. 107, 121110 (2015).

27. N. Vasilantonakis, M. E. Nasir, W. Dickson, G. A. Wurtz, and A. V. Zayats, "Bulk plasmon-polaritons in hyperbolic nanorods," Laser \& Photonics Rev. 9, 345-353 (2015).

28. P. Guo, R. P. Chang, and R. D. Schaller, “Tunable infrared hyperbolic metamaterials with periodic indium-tin-oxide nanorods," Appl. Phys. Lett. 111, 021108 (2017).

29. Z. Jacob and E. E. Narimanov, "Optical hyperspace for plasmons: Dyakonov states in metamaterials," Appl. Phys. Lett. 93, 221109 (2008).

30. A. V. Kildishev, A. Boltasseva, and V. M. Shalaev, "Planar photonics with metasurfaces," Science 339, 1232009 (2013).

31. V. E. Babicheva, "Long-range propagation of plasmon and phonon polaritons in hyperbolic-metamaterial waveguides," J. Opt. 19, 124013 (2017).

32. D. Lu and Z.Liu, "Hyperlenses and metalenses for far-field super-resolution imaging," Nat. Commun. 3, 1205 (2012).

33. C. L. Cortes, W. Newman, S. Molesky, and Z. Jacob, “Quantum nanophotonics using hyperbolic metamaterials," J. Opt. 14, 063001 (2012).

34. I. Avrutsky, I. Salakhutdinov, J. Elser, and V. Podolskiy, "Highly confined optical modes in nanoscale metal-dielectric multilayers," Phys. Rev. B: Condens. Matter Mater. Phys. 75, 242402 (2007).

35. S. V. Zhukovsky, A. A. Orlov, V. E. Babicheva, A. V. Lavrinenko, and J. E. Sipe, "Photonic-band-gap engineering forvolume plasmon polaritons in multiscale multilayer hyperbolic metamaterials," Phys. Rev. A - At. Mol. Opt. Phys. 90, 1-11 (2014).

36. D. Wei, C. Harris, and S. Law, "Volume plasmon polaritons in semiconductor hyperbolic metamaterials," Opt. Mater. Express 7, 2672-2681 (2017).

37. M. Higuchi and J. Takahara, "Plasmonic interpretation of bulk propagating waves in hyperbolic metamaterial optical waveguides," Opt. Express 26, 1918-1929 (2018).

38. S. V. Zhukovsky, O. Kidwai, and J. E. Sipe, "Physical nature of volume plasmon polaritons in hyperbolic metamaterials," Opt. Express 21, 14982-7 (2013).

39. J. Polo and A. Lakhtakia, "Surface electromagnetic waves: A review," Laser Photonics Rev. 5, 234-246 (2011).

40. O. Takayama, A. Bogdanov, and A. V. Lavrinenko, "Photonic surface waves on metamaterials interfaces," J. Physics: Condens. Matter 29, 463001 (2017).

41. J. S. Gomez-Diaz, M. Tymchenko, and A. Alù, "Hyperbolic Plasmons and Topological Transition over Uniaxial Metasurfaces," Phys. Rev. Lett. 114, 233901 (2015).

42. M. I. D'yakonov, "New type of electromagnetic wave propagating at an interface," Sov. Phys. JETP 67, 714-716 (1988).

43. O. Takayama, L.-C. Crasovan, S. K. Johansen, D. Mihalache, D. Artigas, and L. Torner, "Dyakonov Surface Waves: A Review," Electromagnetics 28, 126-145 (2008).

44. O. Takayama, L. Crasovan, D. Artigas, and L. Torner, “Observation of dyakonov surface waves," Phys. Rev. Lett. 102, 043903 (2009).

45. O. Takayama, D. Artigas, and L. Torner, "Practical dyakonons," Opt. Lett. 37, 4311-4313 (2012).

46. D. P. Pulsifer, M. Faryad, and A. Lakhtakia, “Observation of the Dyakonov-Tamm wave,” Phys. Rev. Lett. 111, 243902 (2013).

47. O. Takayama, D. Artigas, and L. Torner, "Lossless directional guiding of light in dielectric nanosheets using Dyakonovsurface waves," Nat. Nanotechnol. 9, 419âĂŞ24 (2014).

48. C. J. Zapata-Rodríguez, J. J. Miret, S. Vuković, and M. R. Belić, "Engineered surface waves in hyperbolic metamaterials," Opt. Express 21, 19113-27 (2013).

49. P. Li, I. Dolado, F. J. Alfaro-Mozaz, F. Casanova, L. E. Hueso, S. Liu, J. H. Edgar, A. Y. Nikitin, S. Vélez, and R. Hillenbrand, "Infrared hyperbolic metasurface based on nanostructured van der Waals materials," Science 359, 892-896 (2018).

50. P. V. Kapitanova, P. Ginzburg, F. J. Rodríguez-Fortuño, D. S. Filonov, P. M. Voroshilov, P. Belov, A. N. Poddubny, Y. S. Kivshar, G. Wurtz, and A. V. Zayats, "Photonic spin Hall effect in hyperbolic metamaterials for polarization-controlled 
routing of subwavelength modes," Nat. Commun. 5, 3226 (2014).

51. D. N. Basov, M. M. Fogler, and F. J. Garcia de Abajo, "Polaritons in van der Waals materials," Science 354, 195 (2016).

52. T. Low, A. Chaves, J. D. Caldwell, A. Kumar, N. X. Fang, P. Avouris, T. F. Heinz, F. Guinea, L. Martin-Moreno, and F. Koppens, "Polaritons in layered 2D materials," Nat. Mater. 16, 182-194 (2016).

53. W. Ma, P. Alonso-González, S. Li, A. Y. Nikitin, J. Yuan, J. Martín-Sánchez, J. Taboada-Gutiérrez, I. Amenabar,P. Li, S. Vélez, C. Tollan, Z. Dai, Y. Zhang, S. Sriram, K. Kalantar-Zadeh, S. T. Lee, R. Hillenbrand, and Q. Bao, "In-plane anisotropic and ultra-low-loss polaritons in a natural van der Waals crystal," Nature 562, 557-562 (2018).

54. S. M. Rytov, "Electromagnetic Properties of Finely Stratified Medium," Sov. Phys. JETP 2, 466-475 (1956).

55. V. M. Agranovich, "Dielectric permeability and influence of external fields on optical properties of superlattices," Solid State Commun. 78, 747-750 (1991).

56. H. Herzig Sheinfux, I. Kaminer, Y. Plotnik, G. Bartal, and M. Segev, "Subwavelength multilayer dielectrics:Ultrasensitive transmission and breakdown of effective-medium theory," Phys. Rev. Lett. 113, 243901 (2014).

57. S. V. Zhukovsky, A. Andryieuski, O. Takayama, E. Shkondin, R. Malureanu, F. Jensen, and A. V. Lavrinenko, "Experimental Demonstration of Effective Medium Approximation Breakdown in Deeply Subwavelength All-Dielectric Multilayers," Phys. Rev. Lett. 115, 177402 (2015).

58. O. Kidwai, S. V. Zhukovsky, and J. E. Sipe, "Effective-medium approach to planar multilayer hyperbolic metamaterials:Strengths and limitations," Phys. Rev. A - At. Mol. Opt. Phys. 85, 1-12 (2012).

59. S. K. Pradhan, B. Xiao, J. R. Skuza, K. Santiago, R. Mundle, and A. K. Pradhan, "Effects of dielectric thickness on optical behavior and tunability of one-dimensional $\mathrm{Ag} / \mathrm{SiO}_{2}$ multilayered metamaterials," Opt. Express 22, 12486-98 (2014).

60. X. Yang, J. Yao, J. Rho, X. Yin, and X. Zhang, "Experimental realization of three-dimensional indefinite cavities at the nanoscale with anomalous scaling laws," Nat. Photon. 6, 450-454 (2012).

61. D. Lu, J. J. Kan, E. E. Fullerton, and Z. Liu, "Enhancing spontaneous emission rates of molecules using nanopatterned multilayer hyperbolic metamaterials," Nat. Nanotechnol. 9, 48-53 (2014).

62. G. V. Naik, B. Saha, J. Liu, S. M. Saber, E. A. Stach, J. M. K. Irudayaraj, T. D. Sands, V. M. Shalaev, and A. Boltasseva, "Epitaxial superlattices with titanium nitride as a plasmonic component for optical hyperbolic metamaterials," Proc. Natl. Acad. Sci. United States Am. 111, 7546-7551 (2014).

63. K. C. Santiago, R. Mundle, C. White, M. Bahoura, and A. K. Pradhan, "Infrared metamaterial by RF magnetron sputtered ZnO/Al:ZnO multilayers," AIP Adv. 8, 035011 (2018).

64. S. Tang, Y. Fang, L. Zho, Z. Liu, and Y. Mei, "Anomalous scaling laws of hyperbolic metamaterials in a tubular geometry,” J. Opt. Soc. Am. B 35, 391-395 (2018).

65. S. S. Kruk, Z. J. Wong, E. Pshenay-Severin, K. O’Brien, D. N. Neshev, Y. S. Kivshar, and X. Zhang, "Magnetic hyperbolic optical metamaterials," Nat. Commun. 7, 11329 (2016).

66. O. Y. Yermakov, A. I. Ovcharenko, M. Song, A. A. Bogdanov, I. V. Iorsh, and Y. S. Kivshar, "Hybrid waves localizedat hyperbolic metasurfaces," Phys. Rev. B 91, 235423 (2015).

67. A. Samusev, I. Mukhin, R. Malureanu, O. Takayama, D. V. Permyakov, I. S. Sinev, D. Baranov, O. Yermakov, I. V. Iorsh, A. A. Bogdanov, and A. V. Lavrinenko, "Polarization-resolved characterization of plasmon waves supportedby an anisotropic metasurface," Opt. Express 25, 32651 (2017).

68. Y. Yang, L. Jing, L. Shen, Z. Wang, B. Zheng, H. Wang, E. Li, N.-H. Shen, T. Koschny, C. M. Soukoulis, and H. Chen, "Hyperbolic spoof plasmonic metasurfaces," NPG Asia Mater. 9, e428 (2017).

69. O. Y. Yermakov, A. A. Hurshkainen, D. A. Dobrykh, P. V. Kapitanova, I. V. Iorsh, S. B. Glybovski, and A. A. Bogdanov, "Experimental observation of hybrid TE-TM polarized surface waves supported by a hyperbolic metasurface," Phys. Rev. B 98, 195404 (2018).

70. E. E. Narimanov, "Photonic hypercrystals," Phys. Rev. X 4, 041014 (2014).

71. V. N. Smolyaninova, B. Yost, D. Lahneman, E. E. Narimanov, and I. I. Smolyaninov, "Self-assembled tunable photonic hyper-crystals,” Sci. Reports 4, 5706 (2014).

72. T. Galfsky, J. Gu, E. E. Narimanov, and V. M. Menon, "Photonic hypercrystals for control of light-matter interactions," Proc. Natl. Acad. Sci. 114, 5125-5129 (2017).

73. X. Liu, J. Gao, H. Yang, X. Wang, S. Tian, and C. Guo, "Hybrid Plasmonic Modes in Multilayer Trench Grating Structures," Adv. Opt. Mater. 2017, 1700496 (2017).

74. X. Liu, J. Gao, J. Gao, H. Yang, X. Wang, T. Wang, Z. Shen, Z. Liu, H. Liu, J. Zhang, Z. Li, Y. Wang, and Q. Li, "Microcavity electrodynamics of hybrid surface plasmon polariton modes in high- quality multilayer trench gratings," Light. Sci. \& Appl. 7, 14 (2018).

75. S. Ishii, M. Y. Shalaginov, V. E. Babicheva, A. Boltasseva, and A. V. Kildishev, "Plasmonic waveguides cladded byhyperbolic metamaterials," Opt. Lett. 39, 4663-4665 (2014).

76. S. Liang, C. Jiang, Z. Yang, D. Li, W. Zhang, T. Mei, and D. Zhang, "Plasmonic slow light waveguide with hyperbolic metamaterials claddings," J. Opt. 20, 065001 (2018).

77. M. Autore, P. Li, I. Dolado, F. J. Alfaro-mozaz, A. Atxabal, F. Casanova, L. E. Hueso, P. Alonso, J. Aizpurua, A. Y.Nikitin, S. Vélez, and R. Hillenbrand, "Phonon-enhanced molecular vibrational spectroscopy at the strong coupling limit," Light. Sci. Appl. 7, 17172 (2017).

78. V. G. Veselago, "The electrodynamics of substances with simultaneously negative values of epsilon and mu," Sov. 
Phys. Uspekhi 10, 509-514 (1968).

79. R. A. Shelby, D. R. Smith, and S. Schultz, "Experimental verification of a negative index of refraction," Science 292, 77-79 (2001).

80. D. R. Smith, J. B. Pendry, and M. C. Wiltshire, "Metamaterials and negative refractive index," Science 305, 788-792 (2004).

81. J. Yao, Z. Liu, Y. Liu, Y. Wang, C. Sun, G. Bartal, A. M. Stacy, and X. Zhang, "Optical negative refraction in bulk metamaterials of nanowires," Science 321, 930 (2008).

82. Z. Jacob, L. V. Alekseyev, and E. Narimanov, "Optical Hyperlens: Far-field imaging beyond the diffraction limit," Opt. Express 14, 8247-8256 (2006).

83. Z. Liu, H. Lee, Y. Xiong, C. Sun, and X. Zhang, "Far-Field Optical Hyperlens Magnifying," Science 315, 1686 (2007).

84. I. I. Smolyaninov, Y.-J. Hung, C. C. Davis, “Magnifying Superlens in the Visible Frequency Range,” Science 315, 1699-1701 (2007).

85. J. Rho, Z. Ye, Y. Xiong, X. Yin, Z. Liu, H. Choi, G. Bartal, and X. Zhang, "Spherical hyperlens for two-dimensional sub-diffractional imaging at visible frequencies," Nat. Commun. 1, 143 (2010).

86. J. Sun and N. M. Litchinitser, "Toward Practical, Subwavelength, Visible-Light Photolithography with Hyperlens," ACS Nano 12, 542-548 (2018).

87. J. Sun, M. I. Shalaev, and N. M. Litchinitser, “ Experimental demonstration of a non-resonant hyperlens in the visible spectral range,” Nat. Commun. 6, 7201 (2015).

88. P. A. Belov, Y. Zhao, S. Sudhakaran, A. Alomainy, and Y. Hao, "Experimental study of the subwavelength imagingby a wire medium slab," Appl. Phys. Lett. 89, 262109 (2006).

89. A. Andryieuski, A. V. Lavrinenko, and D. N. Chigrin, "Graphene hyperlens for terahertz radiation," Phys. Rev. B -Condens. Matter Mater. Phys. 86, 1-5 (2012).

90. T. Repän, A. V. Lavrinenko, and S. V. Zhukovsky, "Dark-field hyperlens: Super-resolution imaging of weakly scattering objects," Opt. Express 23, 25350-25364 (2015).

91. A. Novitsky, T. Repän, S. V. Zhukovsky, and A. V. Lavrinenko, "Subwavelength Hyperlens Resolution With Perfect Contrast Function," Annalen der Physik 530, 1700300 (2018).

92. T. Repän, A. Novitsky, M. Willatzen, and A. V. Lavrinenko, "Pseudocanalization regime for magnetic darkfieldhyperlenses," Phys. Rev. B 96, 195166 (2017).

93. Y. Liu, Y. Ke, H. Luo, and S. Wen, "Photonic spin Hall effect in metasurfaces: A brief review," Nanophotonics 6, 51-70 (2017).

94. X. Ling, X. Zhou, K. Huang, Y. Liu, C.-W. Qiu, H. Luo, and S. Wen, "Recent advances in the spin Hall effect oflight," Rep. Prog. Phys. 80, 066401 (2017).

95. N. B. Baranov, A. Y. Savchenko, and B. Y. Zel'dovich, "Transverse shift of a focal spot due to switching of the signof circular polarization,” JETP Lett. 59, 232-234 (1994).

96. Z. Bomzon, G. Biener, V. Kleiner, and E. Hasman, "Space-variant Pancharatnam-Berry phase optical elementswith computer-generated subwavelength gratings," Opt. Lett. 27, 1141-1143 (2002).

97. K. Y. Bliokh, "Geometrical optics of beams with vortices: Berry phase and orbital angular momentum hall effect," Phys. Rev. Lett. 97, 043901 (2006).

98. K. Y. Bliokh, A. Niv, V. Kleiner, and E. Hasman, "Geometrodynamics of spinning light," Nat. Photonics 2, 748-753 (2008).

99. A. Aiello and J. P. Woerdman, "Role of beam propagation in Goos-Hänchen and Imber-Fedorov shifts," Opt.Lett. 33, 1437-1439 (2008)

100. K. Y. Bliokh and A. Aiello, "Goos-Hänchen and Imbert-Fedorov beam shifts: An overview,” J. Opt. 15, 014001 (2013).

101. H. Chen, S. Zhou, G. Rui, and Q. Zhan, "Magnified photonic spin-Hall effect with curved hyperbolic metamaterials," J. Appl. Phys. 124, 233104 (2018).

102. O. Takayama and G. Puentes, "Enhanced spin Hall effect of light by transmission in a polymer," Opt. Lett. 43, 1343 (2018).

103. O. Y. Yermakov, A. I. Ovcharenko, A. A. Bogdanov, I. V. Iorsh, K. Y. Bliokh, and Y. S. Kivshar, "Spin control oflight with hyperbolic metasurfaces," Phys. Rev. B 94, 075446 (2016).

104. T. Tang, C. Li, and L. Luo, "Enhanced spin Hall effect of tunneling light in hyperbolic metamaterial waveguide," Sci. Reports 6, 30762 (2016).

105. T. Tang, Y. Zhang, J. Li, and L. Luo, "Spin Hall Effect Enhancement of Transmitted Light Through an AnisotropicMetamaterial Slab," IEEE Photonics J. 9, 4600910 (2017).

106. X. Zhou, X. Ling, H. Luo, and S. Wen, "Identifying graphene layers via spin Hall effect of light," Appl. Phys. Lett. 101, 251602 (2012).

107. X. Zhou, L. Sheng, and X. Ling, "Photonic spin Hall effect enabled refractive index sensor using weak measurements," Sci. Reports 8, 1221 (2018).

108. J. Homola, I. Koudela, and S. S. Yee, "Surface plasmon resonance sensors based on diffraction gratings and prismcouplers: sensitivity comparison,” Sensors Actuators, B: Chem. 54, 16-24 (1999).

109. J. Haas and B. Mizaikoff, "Advances in Mid-Infrared Spectroscopy for Chemical Analysis," Annu. Rev. Anal. Chem. 9, 45-68 (2016). 
110. S.-H. Oh and H. Altug, "Performance metrics and enabling technologies for nanoplasmonic biosensors," Nat.Commun. 9, 5263 (2018).

111. R. Adato and H. Altug, "In-situ ultra-sensitive infrared absorption spectroscopy of biomolecule interactions in realtime with plasmonic nanoantennas," Nat.Commun. 4, 2154 (2013).

112. X. Yang, Z. Sun, T. Low, H. Hu, X. Guo, F. J. García de Abajo, P. Avouris, and Q. Dai, "Nanomaterial-BasedPlasmonEnhanced Infrared Spectroscopy," Adv. Mater. 2018, 1704896 (2018).

113. D. Garoli, E. Calandrini, A. Bozzola, M. Ortolani, S. Cattarin, S. Barison, A. Toma, and F. De Angelis, "Boosting infrared energy transfer in 3D nanoporous gold antennas," Nanoscale 9, 915-922 (2017). 